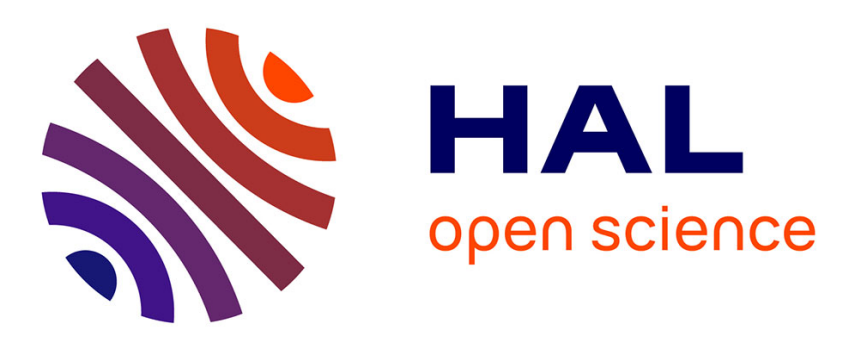

\title{
An optimization formulation of converter control and its general solution for the four-leg two-level inverter
}

\author{
Abdelkader Bouarfa, Marc Bodson, Maurice Fadel
}

\section{To cite this version:}

Abdelkader Bouarfa, Marc Bodson, Maurice Fadel. An optimization formulation of converter control and its general solution for the four-leg two-level inverter. IEEE Transactions on Control Systems Technology, 2018, 26 (5), pp.1901 - 1908. 10.1109/TCST.2017.2738608 . hal-01898582

\section{HAL Id: hal-01898582 \\ https://hal.science/hal-01898582}

Submitted on 18 Oct 2018

HAL is a multi-disciplinary open access archive for the deposit and dissemination of scientific research documents, whether they are published or not. The documents may come from teaching and research institutions in France or abroad, or from public or private research centers.
L'archive ouverte pluridisciplinaire $\mathbf{H A L}$, est destinée au dépôt et à la diffusion de documents scientifiques de niveau recherche, publiés ou non, émanant des établissements d'enseignement et de recherche français ou étrangers, des laboratoires publics ou privés. 


\title{
An optimization formulation of converter con- trol and its general solution for the 4-leg 2-level inverter
}

\author{
Abdelkader Bouarfa, Marc Bodson, Fellow, IEEE, and Maurice Fadel, Member, IEEE
}

\begin{abstract}
The paper proposes an optimization formulation of the control problem for power electronic converters. A benefit of the approach is a systematic method for the control of high switch-count static converters. In the case of the 3-phase, 4-leg, 2level inverter, the framework provides a characterization of all the possible solutions that yield a maximal extension of the inverter linearity range. The method makes it possible to recover wellknown modulation strategies, as well as to discover some new ones having different properties and distinct advantages. The characteristics resulting from different design choices are evaluated in simulations, with consideration being given to the linearity range, total harmonic distortion, and switching losses. Key principles of extension of the proposed method to multilevel, multileg converters are given, as well as motivations for an FPGA-based hardware implementation enabling real-time PWM control.
\end{abstract}

Index Terms-Voltage source inverter, 4-leg 2-level inverter, voltage control, PWM, control allocation, optimization, simplex algorithm, median voltage injection.

\section{INTRODUCTION}

$\mathrm{F}$ IXED high switching-frequency pulse-width modulation (PWM) is an essential class of modulation methods for power converters [1], [2]. For the widely-studied 3-leg 2-level inverter, two popular PWM methods are: (1) carrier-based pulse-width modulation (CBPWM) [1]-[3], using lowfrequency modulating signals and a high-frequency carrier wave, and (2) space vector theory, using a 3-phase geometric vector representation of the inverter and leading to the wellestablished space vector modulation (SVM) techniques [1], [2], [4], [5]. The main theoretical differences between CBPWM and space vector theory are related to the way the degree of freedom left in the inverter is exploited [1], [6]-[12]. Typically, this degree of freedom is used to increase the inverter linearity range [4]-[7], [13], [14], to reduce switching losses [15], [16], or to mitigate current harmonics using suboptimal modulating solutions [17]-[18].

However, the difficulty of developing control strategies in-

A. Bouarfa (corresponding author) and M. Fadel are with LAPLACE, Université de Toulouse, CNRS, INPT, UPS, France: 2 rue Charles Camichel, F-31071 Toulouse, France (bouarfa@laplace.univ-tlse.fr, fadel@laplace.univtlse.fr).

M. Bodson is with University of Utah, Dept. Electrical and Computer Engineering, 50 S Central Campus DR RM 2110, Salt Lake City UT 841129206, USA (bodson@ece.utah.edu). creases rapidly as the number of switches grows, especially with the widely-used space-vector representation. For the 4-leg 2-level inverter, the addition of the fourth-leg raises the number of possible locations of a reference voltage vector from a 2D plane divided into 6 sectors [4], [5] to 24 tetrahedrons in a 3D representation [12], [19]-[21].

Starting from these observations, the paper proposes a new control strategy different from traditional PWM control and based on on-line numerical optimization using linear programming techniques. This strategy is expected to be more generic, to be less dependent on the switch count, and to avoid difficulties encountered with a geometric approach. The control problem of the converter is formulated as an underdetermined constrained optimization problem. Such problem is remarkably similar (although not identical) to the control allocation problem studied previously in flight control and marine applications [22], [23]. In this paper, the focus is placed on the 4-leg 2-level inverter, where a complete characterization of the solutions is possible without the use of numerical techniques, thus enabling a good understanding of the possibilities opened by this new formulation.

A previous implementation of the control allocation approach method for the 4-leg inverter was based on the description of its active voltage vectors [24] and the regular-sampled symmetric PWM (RSPWM) [3]. The correct non-zero vector sequence was computed using a numerical optimization method based on the simplex algorithm, removing the need to identify a reference tetrahedron, as was done in earlier work.

Considering switching cells as available resources subjected to ranges of operation, a different control allocation method is introduced here, reducing the size of the problem considerably. Moreover, the paper proposes an analytical solution of the optimization problem that reduces the control of the inverter to the computation of the median of a special series. Furthermore, $C B P W M$-equivalent formulas are given for modulation laws derived from the new control allocation problem.

As constraints are taken into account in the optimization problem, solutions resulting from control allocation methods for power converters naturally yield a maximal extension of the linearity range of the inverter [24], [25]. Specific choices of the algorithm's parameters produce modulation laws with different properties, all implementable with few computations. Under the general umbrella of the proposed strategy, not only 


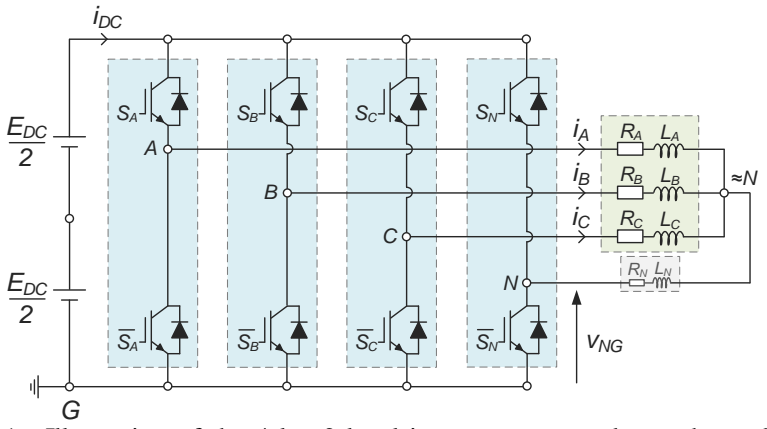

Fig. 1. Illustration of the 4-leg 2-level inverter connected to a three-phase load with impedances $Z_{A}, Z_{B}, Z_{C}$.

are previously-proposed modulation laws found as special cases, but new interesting modulation schemes are discovered as well. Simulation results illustrate the properties of the different methods obtained. Switching losses and total harmonic distortion (THD) on voltages and currents are evaluated in computations for comparison.

\section{4-LEG 2-LEVEL INVERTER ASSUMPTIONS FOR PWM CONTROL}

\section{A. Inverter and load}

The 4-leg 2-level inverter is a well-proven solution for 3phase systems with neutral wire, for applications like distributed generation, active power filtering, common-mode active filtering or fault-tolerant operation of electric drives [12], [19]-[21]. Fig. 1 illustrates the inverter, star-connected to a load with per-phase resistances and inductances values $R_{K}, L_{K}$, $K \in\{A, B, C, N\}$. The load can be unbalanced or nonlinear. The fourth-leg $(N)$ is connected to the neutral point of the load in order to control the neutral voltage and also handle possible unbalanced currents. Here, $R_{N}$ and $L_{N}$ are neglected, and the ground reference $\mathrm{G}$ is the lower DC bus in order to facilitate the derivation of control equations. $E_{D C}$ is the DC bus voltage.

\section{B. Switching cells}

A switching cell is taken to be an association of two functional switches working at complementary binary states. Dead times are neglected. Each leg is comprised of one switching cell. For $K \in\{A, B, C, N\}$, the state of the switching cell $K$ is the state of the corresponding upper switch, $S_{K}$, equal to 0 when the switch is off and 1 when the switch is on.

\section{Output voltages, load voltages}

The 4 output voltages $V_{K G}$ are referred to the ground $G$. Regarding the star-connection, it is useful to define for each of the three first legs a relative switching state $S_{K N}=S_{K}-S_{N}$. Then, the 3 independent load voltages, referred to the neutral point $N$, are given by $V_{K N}=E_{D C} S_{K N}$, for $K \in\{A, B, C\}$.

\section{Pulse-width modulation}

The mean value $D_{K}=\left\langle S_{K}\right\rangle_{T s}$ of $S_{K}$ over the switching period $T_{S}$ defines the duty cycle of the switching cell $K, D_{K} \in[0,1]$. Mean-value references voltages are obtained with RSPWM by determining the gating pulses $S_{K}$ from the duty cycles $D_{K}$ as illustrated on Fig. 2. The load voltage mean values are

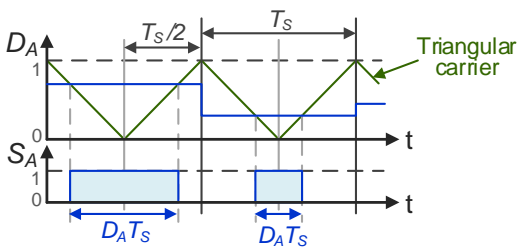

Fig. 2. Illustration of the realization of gating pulse on switch A of width $D_{A} \cdot T_{S}$ and centered over the switching period $T_{S}$ (RSPWM).

$\forall K \in\{A, B, C\}, \quad<V_{K N}>_{T_{S}}=E_{D C}\left(D_{K}-D_{N}\right)=E_{D C} D_{K N}$

The linearity range of the converter is defined here as the range of amplitude of the 3-phase sinusoidal reference voltage system for which the converter delivers a voltage system with the desired values. Because of the star-connection, with classic sinusoidal PWM (SPWM), the linearity range is restricted to $E_{D C} / 2$. A common modification extends this range by varying the neutral potential of the load. When the desired voltages are balanced and three-phase, the maximal output amplitude achievable linearly is $1 / \sqrt{3} \cdot E_{D C}$, as for SVM [4], [5] or SPWM with 1/6 third-harmonic injection (THIPWM1/6) [13], [14].

\section{CONTROL ALLOCATION FOR INVERTER CONTROL}

\section{A. Control allocation methods}

Control allocation methods were developed in aerospace problems as a solution for over-actuated systems subjected to constraints [22], [23]. It is assumed that the process to be controlled can be modeled by a constrained system of $m$ control equations with $n$ unknowns, with $m<n$ :

$$
B \cdot u=a, \quad u_{\min } \leq u \leq u_{\max }
$$

The matrix $B$, of size $m \times n$, specifies the effectiveness of the actuators. $B$ is assumed to be of rank $m$ (rows are linearly independent). The vector $u$, of length $n$ (column), is the control vector that specifies the chosen use of actuators. The vector $a$, of length $m$ (column), is the resulting output vector. Denote $u_{\min }$ and $u_{\max }$ (vectors) as the minimum and maximum bounds of each component of the control vector $u$, respectively. One wants to find a control vector $u$ to obtain a given desired output vector $a_{d}$. Considering the constraints, the problem (2) may have zero, one, or an infinity of solutions. Methods have been developed to extract a solution from the whole set of feasible solutions by formulating an optimization problem.

The objective of the 4-leg 2-level inverter control is to obtain a desired reference vector $a_{d}=V_{\text {ref }}=\left(V_{A N r e f}, V_{B N r e f}, V_{C N r e f}\right)^{T}$ of 3 load voltages on average over the switching period $T_{S}$. In order to scale the voltages with respect to $E_{D C}$, define the scaled reference vector as $\Delta D_{r e f}=V_{r e f} / E_{D C}=\left(D_{A N r e f}, D_{B N r e f}\right.$, $\left.D_{C N r e f}\right)^{T}$. Here, $\Delta D_{\text {ref }}$ is similar to the difference of duty cycles between the three first legs and the fourth one, see (1). Then, the system of equations of (2) depends on the chosen control variables and the converter control requirements.

\section{B. Switching cell formulation}

The control problem can be formulated as finding duty cycle 
commands $D_{S}$ to the cells such that

$$
\begin{gathered}
\Delta D_{r e f}=M_{S} D_{S}, \quad 0 \leq D_{S} \leq 1 \\
M_{S}=\left(\begin{array}{llll}
1 & 0 & 0 & -1 \\
0 & 1 & 0 & -1 \\
0 & 0 & 1 & -1
\end{array}\right) \quad D_{S}=\left(\begin{array}{llll}
D_{A} & D_{B} & D_{C} & D_{N}
\end{array}\right)^{T}
\end{gathered}
$$

The switching cell formulation (3) is a simpler alternative to the space vector formulation in [24], introducing less control variables and a smaller matrix. In order to find a control solution to the under-determined constrained control problem (3), the following optimization problem is defined:

$$
\begin{gathered}
\min _{D_{S}} J, \quad J=\left\|M_{S} \cdot D_{S}-\Delta D_{r e f}\right\|_{1}+\varepsilon_{0} \cdot \varepsilon \cdot\left|D_{S}-D_{S \text { pref }}\right| \\
\text { subject to } 0 \leq D_{S} \leq 1
\end{gathered}
$$

The primary criterion $J_{c t r l}\left(D_{S}\right)=\left\|M_{S} D_{S}-\Delta D_{\text {ref }}\right\|_{1}$ is a control error based on the control equation in (3). Because the solution is often not unique, the secondary criterion $J_{\text {pref }}\left(D_{S}\right)=$ $\varepsilon \cdot\left|D_{S}-D_{\text {Spref }}\right|$ is a deviation error added to specify preferred uses of the switches, where || denotes the application of the element-wise absolute value. The column vector $D_{\text {Spref }}=$ $\left(D_{\text {Apref }}, D_{\text {Bpref }}, D_{\text {Cpref }}, D_{\text {Npref }}\right)^{T}$ corresponds to the preferred duty cycle values. The row vector $\varepsilon=\left(\varepsilon_{A}, \varepsilon_{B}, \varepsilon_{C}, \varepsilon_{N}\right)$ helps to give further preference to one or more switches, which makes it possible to affect the weight of deviation errors from preferred positions in $J_{\text {pref. }}$. Although the parameters $\varepsilon_{K}$ can be real numbers, we will restrict them to be integers, with little loss of generality. This choice will result in a simple solution of the optimization problem. Finally, the small real parameter $\varepsilon_{0}$ gives priority to the minimization of the control error $J_{\text {ctrl }}$ [22].

The optimal solutions are determined by the choice of the parameters $D_{\text {Spref }}$ and $\varepsilon$, which produce a large number of possible configurations. We will discuss a few choices in the next sections. The associated control diagram is shown on Fig. 3.

\section{CHARACTERIZATION OF ALL FEASIBLE SOLUTIONS WHEN THE REFERENCE IS ACHIEVABLE}

\section{A. Constraint set when the reference is achievable}

When the reference vector is achievable, the optimization problem can be simplified. Solutions can be determined analytically, depending on the parameters $D_{\text {Spref, }}$, and $\varepsilon$. This leads to relatively simple expressions of the modulating signals.

Due to the particular form of $M_{S}$, the solutions that achieve the reference can be characterized by the single variable $D_{N}$ :

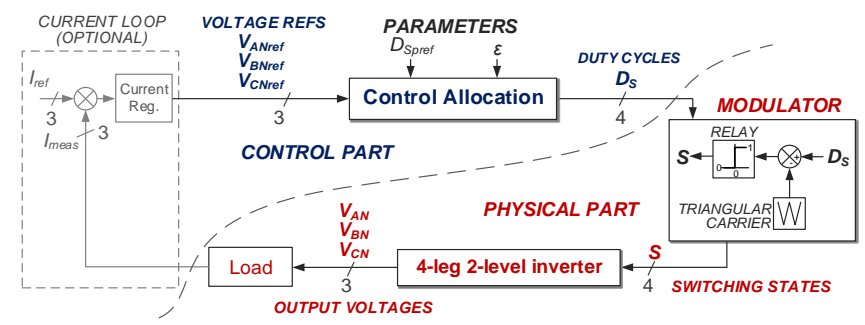

Fig. 3. Diagram of the proposed control allocation method.

$$
\forall K \in\{A, B, C\}, \quad D_{K}=D_{K N_{r e f}}+D_{N}
$$

If there is at least one positive and one negative voltage in the reference vector (it is the case if the reference voltage vector is three-phase), bounds $D_{N \min }$ and $D_{N \max }$ can be defined as

$$
D_{N_{\text {min }}}=-\min \Delta D_{r e f}, \quad D_{N_{\max }}=1-\max \Delta D_{r e f}
$$

and the whole set of feasible solutions that achieve the reference vector is described by constraints on $D_{N}$ alone:

$$
\forall D_{N} \in\left[D_{N_{\min }}, D_{N_{\max }}\right], J_{c t r l}\left(D_{N}\right)=0
$$

Therefore, as long as the constraint set is not empty, i.e., as long as $D_{N \min } \leq D_{N \max }$, any signal resulting from solutions of (5) will achieve a maximal linearity range.

\section{B. Reduction of the solution to a single decision variable}

The secondary criterion to be minimized is

$$
J_{\text {pref }}\left(D_{S}\right)=\sum_{K \in\{A, B, C, N\}} \varepsilon_{K}\left|D_{K}-D_{K \text { pref }}\right|
$$

By defining a series $d_{\bmod }$ of elements $d_{\bmod K}$ as

$$
\begin{array}{cc}
\forall K \in\{A, B, C\}, & d_{\bmod K}=D_{K \text { pref }}-D_{K N \text { ref }} \\
d_{\text {mod } N}=D_{N_{\text {pref }}} \quad d_{\text {mod }}=\left(d_{\bmod K}\right)_{K \in\{A, B, C, N\}}
\end{array}
$$

and by substitution in (25), the criterion $J_{\text {pref }}$ can be now reformulated as an explicit function of $D_{N}$ :

$$
J_{\text {pref }}\left(D_{N}\right)=\sum_{K \in\{A, B, C, N\}} \varepsilon_{K}\left|D_{N}-d_{\bmod K}\right|
$$

that is a sum of distances on the real-axis from $D_{N}$ to the points $d_{\bmod K}$ and weighted by the parameters $\varepsilon_{K}$. The single decision variable $D_{N}$ solely represents the available functional degree of freedom. The optimization problem is considerably simplified.

\section{Solution of the optimization problem}

The minimization of sums of weighted absolute deviations from a unique number (here $D_{N}$ ) to other fixed numbers (here $\left.d_{\bmod K}\right)$ is a well-known mathematical problem [26]-[30]. Here, the solution is introduced thanks to the definition of the special series $d_{\bmod \varepsilon}$, for which each $d_{\bmod K}$ appears $\varepsilon_{K}$ times. From [26][30], it can be deduced that, without considering constraints, the solution of the minimization of (13) reduces to the determination of the median (denoted med) of the series $d_{\bmod \varepsilon}$ for all values of the design parameters.

The sum of all the $\varepsilon_{K}$ gives the size (or number of elements) of $d_{\text {mod } \varepsilon}$. In practice, two distinct cases are encountered: (1) the series $d_{\text {mod }}$ is of odd size: the optimal value of $D_{N}$ denoted $D_{N o p t}$ is the midpoint of the series and is unique; (2) the series $d_{\bmod \varepsilon}$ is of even size: $D_{N o p t}$ is a set of values of $D_{N}$ belonging to the segment delimited by the two midpoints of the series.

Fig. 4 illustrates the optimal solutions depending on the nature of the size of $d_{\bmod \varepsilon}$. The criterion $J_{\text {pref }}$ is a piecewise linear function due to the use of absolute values. The "even" case in Fig. 4 (a) is inconvenient because there is an infinite number of solutions, and control is not uniquely determined. In practice, a 
slight adjustment of the problem statement is sufficient to make the optimal solution unique, as shown in Fig. 4 (b).

Finally, the median set of the series $d_{\bmod \varepsilon}$ has to be constrained by the bounds $D_{N \min }$ and $D_{N \max }$ in order to obtain feasible solutions. Therefore, optimal solutions of the constrained problem are the elements $D_{\text {Nopt }}$ belonging to the intersection of the median set of $d_{\bmod \varepsilon}$ and of the segment [ $\left.D_{N \min }, D_{N \max }\right]$.

\section{Special choices of parameters}

The choice of parameters $D_{\text {Spref }}$ and $\varepsilon$ has a direct and significant effect on the nature of $d_{\bmod \varepsilon}$, on the optimal set of $D_{N}$, and on the resulting modulation strategy. Sets of parameters having interesting practical implications are given as examples.

The adjustment of $D_{\text {Spref }}$ translates into preferred duty cycle values of for each switch. The most common choices for $D_{\text {Spref }}$ would be $D_{\text {Spref }} \in\left\{(0 \ldots 0)^{T},(1 \ldots 1)^{T},(0.5 \ldots 0.5)^{T}\right\}$.

The first obvious choice of $\varepsilon$ is a vector of ones, implying no discrimination between switches. More generally, to avoid non-symmetric behavior with respect to the three phases, the first three weights should be identical. This yields the following propositions for adjusting $\varepsilon:\left\{\left(\begin{array}{lll}1 & \ldots\end{array}\right),\left(\begin{array}{llll}1 & 1 & 1 & 0\end{array}\right),\left(\begin{array}{llll}0 & 0 & 0 & 1\end{array}\right)\right\}$.

\section{EXAMPLES \& SIMULATION RESULTS}

Specific solutions are derived and illustrated for the most interesting choices of parameters $D_{\text {Spref }}$ and $\varepsilon$, as summarized in Table I. Simulations are carried out in the MATLAB Simulink environment. Evaluations of switching losses, THD on voltages and THD on currents are given later for comparison.

For the following figures showing duty cycles, the scaled reference amplitude value increases from left (a) to right (c) until the limit of $1 / \sqrt{ } 3$ is reached. A whole fundamental period is illustrated. Reference voltages are chosen to be three-phase and balanced for simplicity, but the method also works with unbalanced references. As it will be useful for determining optimal solutions, we denote $\Delta D_{\text {med }}=$ med $\Delta D_{\text {ref }}$ the median of the scaled reference vector.

\section{A. Configuration 1}

Selected configuration: First of all, consider the natural choice where all preferred duty cycles are set to 0.5 , which

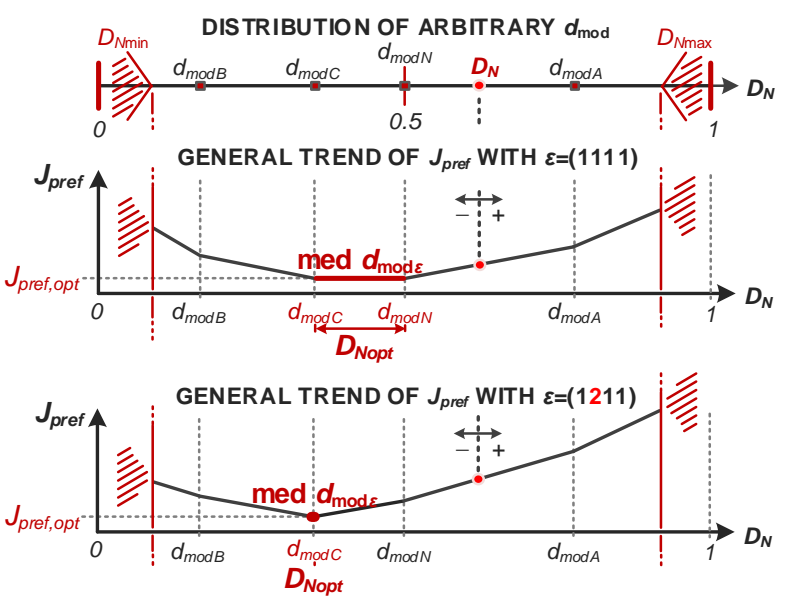

Fig. 4. Illustration of the effect of parameters on the solving of the simplified optimization problem with arbitrary $\Delta D_{\text {ref. }} D_{\text {Spref }}=\left(\begin{array}{llll}0.5 & 0.5 & 0.5 & 0.5\end{array}\right)^{T}$. means $D_{\text {Spref }}=\left(\begin{array}{llll}0.5 & 0.5 & 0.5 & 0.5\end{array}\right)^{T}$ and $\varepsilon=\left(\begin{array}{llll}1 & 1 & 1 & 1\end{array}\right)$.

Determination of the median: As all the $\varepsilon_{K}$ are identical, all the $d_{\bmod K}$ have the same occurrence in the series $d_{\bmod \varepsilon}$ :

$$
d_{\text {mod } \varepsilon}=\left\{0.5-D_{A N \text { ref }}, 0.5-D_{B N \text { ref }}, 0.5-D_{C N \text { ref }}, 0.5\right\}
$$

The size is even. Therefore, optimal solutions $D_{N \text { opt }}$ belong to the segment defined by the two midpoints of $d_{\bmod \varepsilon}$. In the case where the reference system is three-phase, 0.5 is necessarily one of the two midpoints of $d_{\bmod \varepsilon}$. The second midpoint is linked to $\Delta D_{\text {med. }}$. Consequently,

$$
\operatorname{med} d_{\bmod \varepsilon}=\left[0.5-\Delta D_{\mathrm{med}}, 0.5\right]
$$

Remark: If $\Delta D_{\text {med }}$ is negative, the segment [0.5- $\left.\Delta D_{\text {med }}, 0.5\right]$ has to be replaced by $\left[0.5,0.5-\Delta D_{\text {med }}\right]$. For simplicity, this trivial adjustment will be assumed to be made if necessary.

Optimal solutions: Finally, optimal solutions are any value belonging to $\left[0.5-\Delta D_{\text {med }}, 0.5\right]$ and saturated by $\left[D_{N \min }, D_{N \max }\right]$.

Fig. 5 gives an illustration of the domain of the $D_{N \text { opt }}$ waveforms. The bounds $D_{N \min }$ and $D_{N \max }$ are represented by the extremal bold lines, delimiting the saturation domain. As the reference amplitude increases, the saturation domain becomes narrower. The scaled value of $1 / \sqrt{ } 3$ is the limit where for some instants $\theta=k \pi / 3, k \in\{0, \ldots, 6\}$, the two extremal bold lines meet, meaning that $D_{N \min }$ is equal to $D_{N \max }$, see Fig. 5 (c).

In Fig. 5, the two plain midlines draw the bounds of the optimal domain of $D_{N}$ (hatched area). As the scaled amplitude increases, the median segment grows wider and eventually encounters the saturation domain (from (b)). The two lines labelled (1) and (2) correspond to the maximum and minimum bounds of the nonsaturated optimal solution set, respectively.

Fig. 6 shows the optimal domain of the $D_{A}$ waveforms, determined from the optimal domain of $D_{N}$ by using (6). The fundamental wave is represented by the dashed gray midline (when visible). As for Fig. 5, the optimal domain of $D_{A}$ obtained if $D_{N}$ 's is not saturated is represented by the lines labelled (1) and (2). The parts of any optimal saturated $D_{N}$ modulating signal that encounters the bounds $D_{N \min }$ or $D_{N \max }$ correspond to periods where a leg is continuously clamped to the DC bus or to the ground, as observed on Fig. 6 (b)-(c).

When $D_{N}$ is not saturated, a certain regular waveform of $D_{A}$ is identified, which stays proportional to the reference amplitude until saturation appears. Then, as a result of the saturation, the optimal domain of $D_{A}$ changes form compared to Fig. 6 (a) in order to achieve the correct fundamental wave. This particular property is shared by most of modulation strategies obtained from the proposed control allocation method.

TABLE I

\begin{tabular}{|c|c|c|c|}
\hline$n^{\circ}$ & $D_{\text {Spref }}^{T}$ & $\varepsilon$ & Resulting PWM laws \\
\hline 1 & $\left(\begin{array}{llll}0.5 & 0.5 & 0.5 & 0.5\end{array}\right)$ & $\left(\begin{array}{llll}1 & 1 & 1 & 1\end{array}\right)$ & undetermined \\
\hline 2 & $\left(\begin{array}{llll}0.5 & 0.5 & 0.5 & 0.5\end{array}\right)$ & $\left(\begin{array}{llll}1 & 1 & 1 & 0\end{array}\right)$ & OMIPWM [25] \\
\hline 3 & $\left(\begin{array}{llll}0.5 & 0.5 & 0.5 & 0.5\end{array}\right)$ & $\left(\begin{array}{llll}0 & 0 & 0 & 1\end{array}\right)$ & ASPWM \\
\hline 4 & $\left(\begin{array}{llll}1 & 1 & 1 & 1\end{array}\right)$ & $\left(\begin{array}{llll}1 & 1 & 1 & 1\end{array}\right)$ & DPWMmax [1], [9], [11] \\
\hline$\sim$ & $\left(\begin{array}{llllll}0 & 0 & 0 & 0\end{array}\right)$ & $\left(\begin{array}{llll}1 & 1 & 1 & 1\end{array}\right)$ & DPWMmin [1], [9], [11] \\
\hline
\end{tabular}

CONFIGURATION EXAMPLES OF THE CONTROL ALLOCATION METHOD

OMIPWM: opposite median-voltage injection PWM; ASPWM: adaptive sinus PWM; DPWM: discontinuous PWM. 


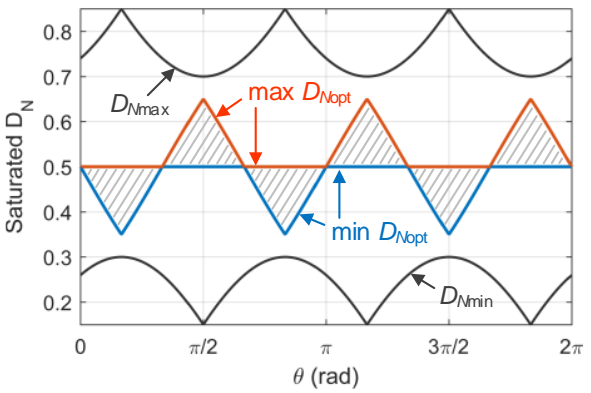

(a) $V_{\text {ref }}=0.3 E_{D C}$

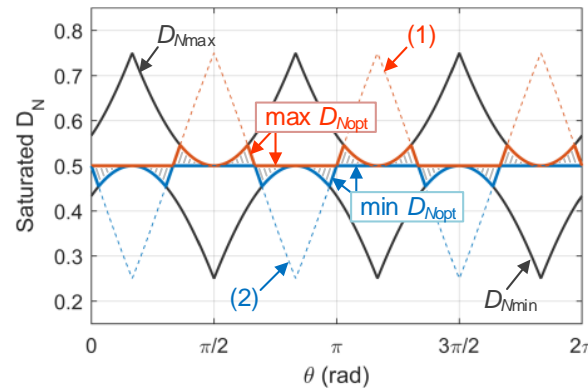

(b) $V_{\text {ref }}=0.5 E_{D C}$

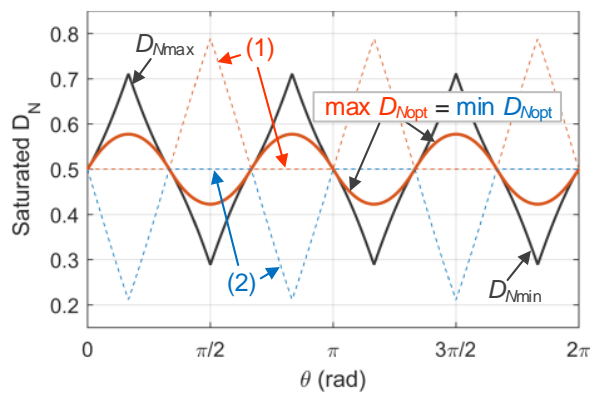

(c) $V_{\text {ref }}=1 / \sqrt{ } 3 E_{D C}$

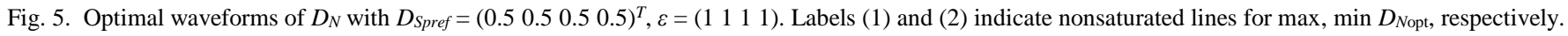

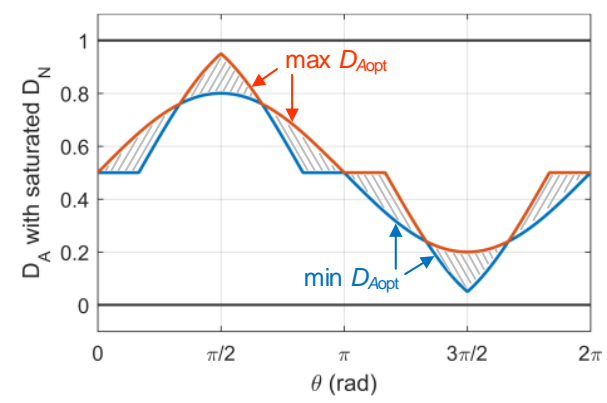

(a) $V_{\text {ref }}=0.3 E_{D C}$

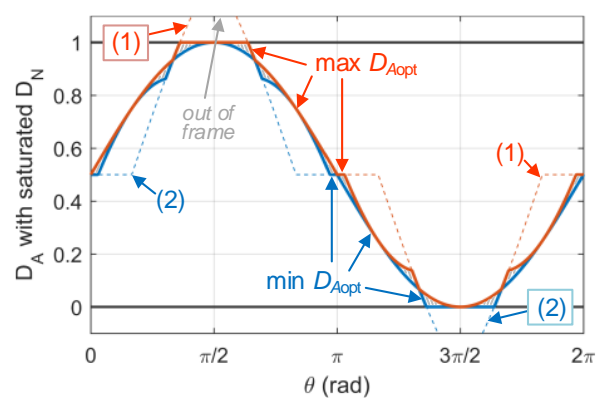

(b) $V_{\text {ref }}=0.5 E_{D C}$

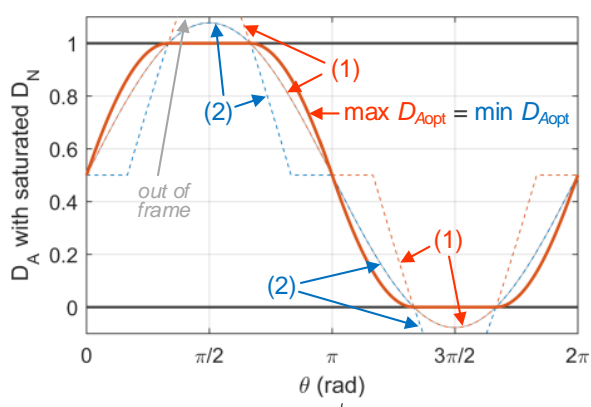

(c) $V_{\text {ref }}=1 / \sqrt{ } 3 E_{D C}$

Fig. 6. Optimal waveforms of $D_{A}$ with $D_{\text {Spref }}=\left(\begin{array}{lllll}0.5 & 0.5 & 0.5 & 0.5\end{array}\right)^{T}, \varepsilon=\left(\begin{array}{llll}1 & 1 & 1 & 1\end{array}\right)$. Labels (1) and (2) indicate nonsaturated lines for max, min $D_{A o p t}$, respectively.

\section{B. Configuration 2}

Selected configuration: With the previous configuration, the optimal solution was not unique. In order to force the series to be of odd cardinal, a first possibility is to remove the preference on the fourth duty cycle that controls the neutral potential while keeping the symmetry between the three phases. This leads to $D_{\text {Spref }}=\left(\begin{array}{llll}0.5 & 0.5 & 0.5 & 0.5\end{array}\right)^{T}$ and $\varepsilon=\left(\begin{array}{llll}1 & 1 & 1 & 0\end{array}\right)$.

Determination of the median: $d_{\bmod N}$ does not appear in the series $d_{\text {mod } \varepsilon}$ and, as the other $\varepsilon_{K}$ are identical,

$d_{\bmod \varepsilon}=\left\{0.5-D_{A N \text { ref }}, 0.5-D_{B N_{\text {ref }}}, 0.5-D_{C N \text { ref }}\right\}$

Thus, the median is directly given by med $d_{\bmod \varepsilon}=0.5-\Delta D_{\text {med }}$.

Optimal solution: the optimal value $D_{N o p t}$ is unique and is equal to $0.5-\Delta D_{\text {med }}$ saturated by [ $\left.D_{N \min }, D_{N \max }\right]$. It corresponds to parts of the optimal domain given by the previous configuration and farthest from 0.5 in Fig. 5. Thanks to the analytical solution, a carrier-based equivalent method can be given. It consists in injecting the opposite of the median reference in the waveform of $D_{N}$ as a zero-sequence signal. This new modulation method was recently proposed in [25] under the name of opposite median-voltage injection PWM (OMIPWM). Unlike SVM, where the zero-sequence signal always corresponds to the value of the midpoint between $D_{N \min }$ and $D_{N \max }$ (see [6], [8]-[11], [16], [25]), $D_{N}$ will tend to reach the bounds $D_{N \min }$ and $D_{N \max }$ for OMIPWM. From a certain reference amplitude value, discontinuous modulation appears with increasing discontinuous periods. This feature is highlighted in Fig. 5 (b)-(c) at $\theta=\pi / 6+k \pi / 3, k \in\{0, \ldots, 5\}$. OMIPWM is a new method, easy to implement, which automatically ensures the transition from a continuous to a discontinuous modulation scheme as a function of the reference amplitude [25].

\section{Configuration 3}

Selected configuration: another possibility is to place a preference for the fourth duty cycle at 0.5 , which means keeping the neutral potential nearest to half of the DC bus voltage: $D_{\text {Spref }}=\left(\begin{array}{llll}0.5 & 0.5 & 0.5 & 0.5\end{array}\right)^{T}$ and $\varepsilon=\left(\begin{array}{llll}0 & 0 & 0 & 1\end{array}\right)$.

Determination of the median: This time, only $\varepsilon_{N}$ is non-null. $d_{\bmod N}=0.5$ is the only element of $d_{\bmod \varepsilon}$, thus, med $d_{\bmod \varepsilon}=0.5$.

Optimal solution: $D_{N \text { opt }}$ is unique and corresponds to the value 0.5 saturated by $\left[D_{N \min }, D_{N \max }\right]$.

Fig. 7 shows the resulting optimal waveform of $D_{N}$. In fact, most of the time, the optimal solution corresponds to SPWM, as shown on Fig. 8. However, SPWM has a linearity range limited to the scaled amplitude value of 0.5 . As can be deduced from Fig. 7 (b), the constant $D_{N}$ solution value of 0.5 starts to be saturated by the bounds $D_{N \min }$ and $D_{N \max }$. It gives the minimal adjustment of the neutral potential needed to achieve the reference. A benefit of the optimal solution given by configuration 3 is that, unlike for SPWM, the maximal limit of $1 / \sqrt{3}$ is reached. It appears that this solution has not been proposed before in the literature. The associated modulation law is called here adaptive sinus PWM (ASPWM).

\section{Configuration 4}

Selected configuration: it is also possible to adjust $D_{\text {Spref }}$ in order to specify a preferred value of duty cycle. For configuration 4, we set $D_{\text {Spref }}=\left(\begin{array}{llll}1 & 1 & 1 & 1\end{array}\right)^{T}$ and $\varepsilon=\left(\begin{array}{llll}1 & 1 & 1 & 1\end{array}\right)$.

Determination of the median: the median of the series med $d_{\bmod \varepsilon}$ is not reduced to a unique element. It corresponds to $\left[1-\Delta D_{\text {med, }}, 1\right]$ and it is always out of the saturation domain.

Optimal solution: With the selected vector $D_{\text {spref }}$, the optimal solution is $D_{N \max }$, whatever the reference amplitude value is (of course comprised between 0 and $1 / \sqrt{ } 3$ ). This solution 


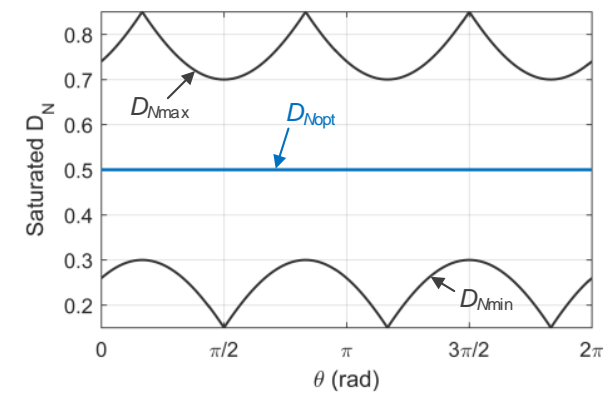

(a) $V_{r e f}=0.3 E_{D C}$

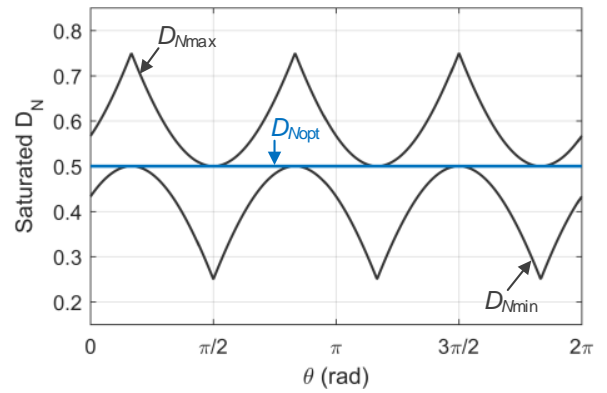

(b) $V_{r e f}=0.5 E_{D C}$

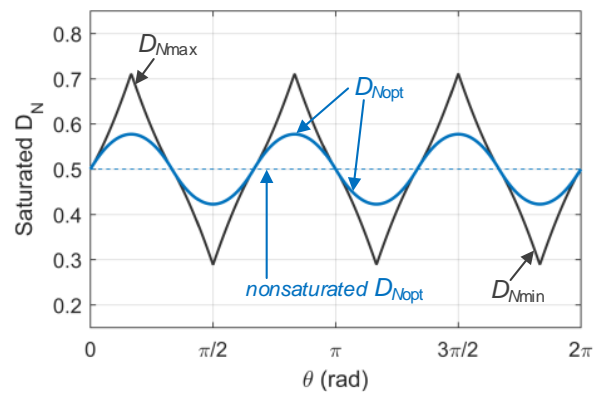

(c) $V_{r e f}=1 / \sqrt{3} E_{D C}$

Fig. 7. Optimal waveforms of $D_{N}$ with $D_{S p r e f}=\left(\begin{array}{llll}0.5 & 0.5 & 0.5 & 0.5\end{array}\right)^{T}, \varepsilon=\left(\begin{array}{llll}0 & 0 & 0 & 1\end{array}\right)$, corresponding to ASPWM.

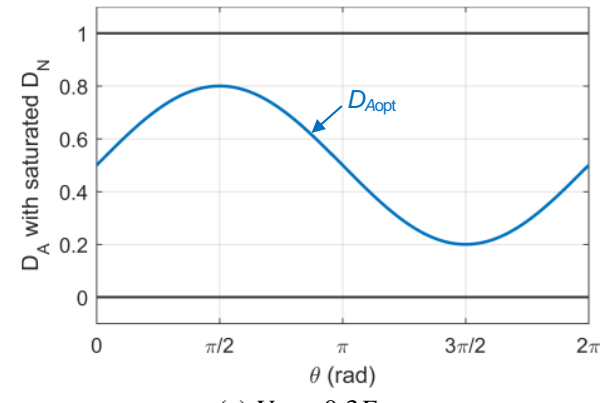

(a) $V_{\text {ref }}=0.3 E_{D C}$

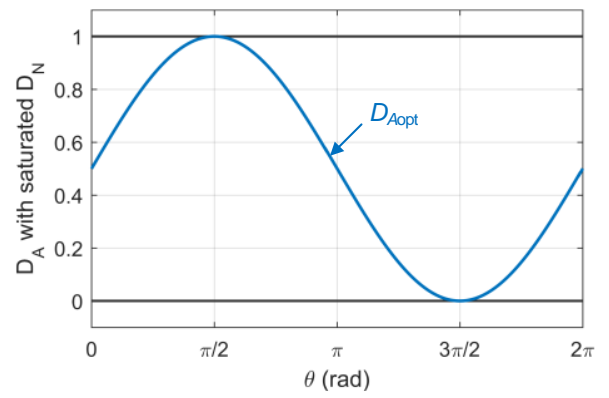

(b) $V_{r e f}=0.5 E_{D C}$

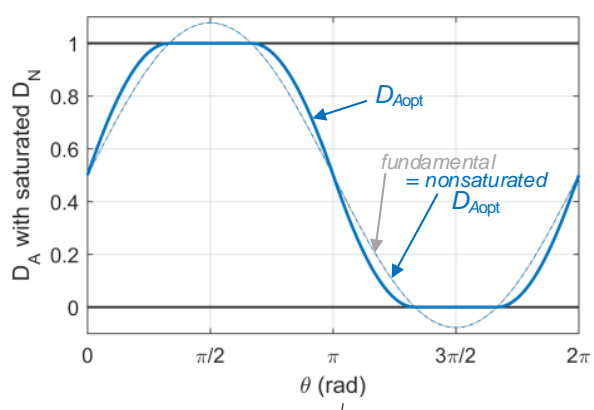

(c) $V_{r e f}=1 / \sqrt{ } 3 E_{D C}$

Fig. 8. Optimal waveforms of $D_{A}$ with $D_{\text {Spref }}=\left(\begin{array}{llll}0.5 & 0.5 & 0.5 & 0.5\end{array}\right)^{T}, \varepsilon=\left(\begin{array}{llll}0 & 0 & 0 & 1\end{array}\right)$, corresponding to ASPWM.

corresponds to the DPWMmax technique [1], [8]-[11], [16].

The same reasoning can be carried out with a preferred duty cycle value of 0 . This choice leads to the optimal solution $D_{N \min }$, which corresponds to the DPWMmin technique.

\section{E. Comparison of performances}

Performance criteria of importance to power electronic converters were evaluated in simulations to gain a better understanding of the differences between the various solutions. Simulation parameters are given in Table II.

Basic estimation of switching-loss energies over the simulation time were obtained by using simple parametric models derived from curves available on datasheets. Only general trends should be considered. Switching losses are shown in Fig. 9 (a). Two quasi-parallel lines, one higher than the other, describe the evolution of the switching losses, for SVM and for DPWMmin or DPWMmax. The trend for OMIPWM is remarkable since the curve follows the SVM curve, and then switches to the DPWM curve as a function of the amplitude [25]. It is also the case for ASPWM, but the transition occurs for a much greater reference signal, because ASPWM is equivalent to SPWM until the scaled value of 0.5 is reached.

Fig. 9 (b) shows the evolution of the THD on the load voltage $V_{A N}$. THD was evaluated using the function thd of the Signal Processing Toolbox in MATLAB. THD trends on voltages are very similar for all methods, but some differences are found for currents that highlight differences in the spectral distributions, see in Fig. 9 (c). As for switching losses, only general trends should be considered since the precise value of THD depends on the load impedances. The transition effect of OMIPWM is also observed with the THD on the currents. At low amplitudes, the THD for OMIPWM on the currents is higher than SVM, but fairly close compared to the THD for the DPWM's. As the amplitude increases, the THD on currents for OMIPWM converges to DPWM THD's, which have dropped and are closer to the SVM THD's. The same trend is found for ASPWM, but in a more mitigated manner, as ASPWM remains a continuous PWM method most of the time.

OMIPWM presents better harmonic quality at low reference amplitude and lower switching losses at high reference amplitude. It constitutes an advantageous compromise between continuous and discontinuous modulation [25]. ASPWM has the particularity of moving the neutral potential as little as possible from half the DC voltage, and moreover of not having an effect on the neutral potential when not needed.

\section{EXTENSION TO MULTILEVEL, MULTILEG CONVERTERS}

In this paper, we introduced a new control allocation method for the voltage control of the 4-leg 2-level inverter. However, the general approach is also applicable to multilevel, multileg converters, as long as one derives control equations linking duty cycles of switching cells to desired outputs. As the numerical optimization is based on the simplex algorithm, control equations must be linear (or linearized).

We already developed control allocation methods for the 3phase multilevel flying capacitor inverter [31] and the 3-phase modular multilevel inverter [32]. To ensure safe and efficient operation of these converters, additional issues must be addressed, like the active-balancing of quantities related to energy-storing elements, like capacitor voltages. Linear discrete control equations can be derived from differential equations of capacitor voltages and/or output currents by sampling and holding state values and by performing 1-order predictions. Then, a LP problem is formulated. The problem is updated and solved for each control period. As a result of the optimized control, the method in [31] led to a fast active-balancing of 


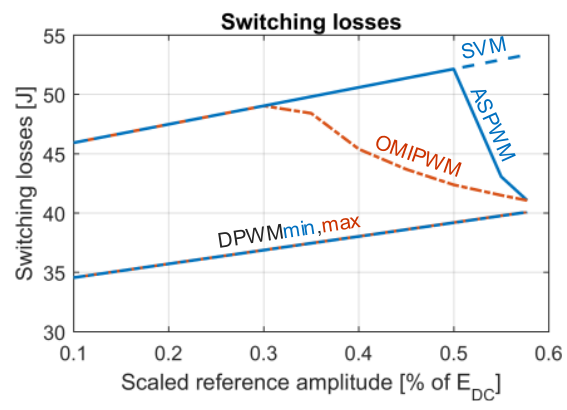

(a)

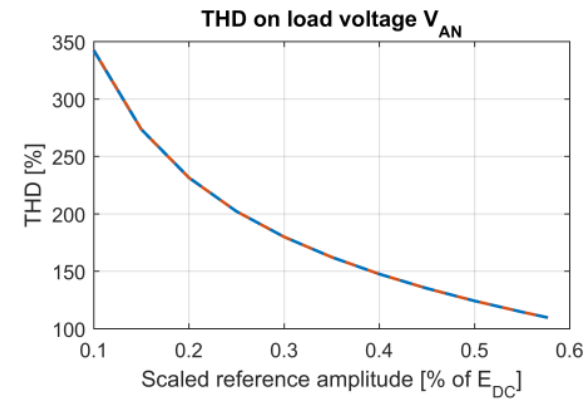

(b)

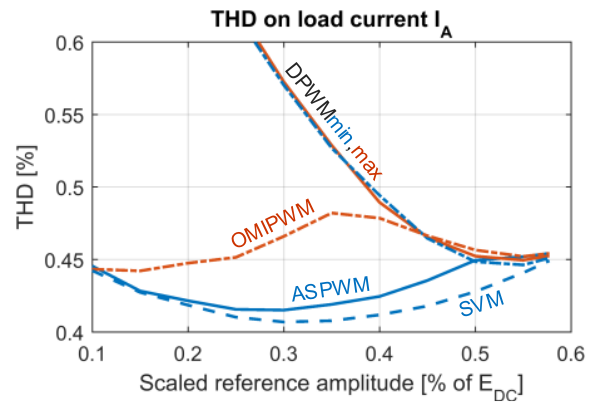

(c)

Fig. 9. Performance evaluation in simulation for proposed configurations. (a) Switching losses. (b) THD on load voltage $V_{A N}$. (c) THD on load current $I_{A}$.

capacitor voltages. Finally, 4-leg inverters are already multileg inverters, but, in the same way, the approach is expected to be also applicable to converters with more than 4 legs.

\section{ABOUT THE HARDWARE IMPLEMENTATION OF THE CONTROL ALLOCATION METHOD}

To achieve an efficient PWM operation, a high control frequency is needed. Typical switching periods are around few hundreds of microseconds. FPGA implementation is a solution for complying with these strong time constraints, thanks to short memory read/write access times and advantages of hardwired logic like parallelism and pipelining. Up to now, the problem of the FPGA implementation of the simplex algorithm has been rarely addressed, and the first implementation known to the authors was proposed in 2006 [33] with 18-bit data precision. It led to a gain in computing times up to 20 times those obtained with optimized commercial solvers on PC's [33].

We are currently working on an FPGA implementation of the simplex algorithm dedicated to control allocation methods for power converters, using a Terasic TR4 development board (Altera Stratix IV FPGA core), with 32-bit floating-point data representation. A first validation of our implementation was carried out with small-size arbitrary linear programs thanks to the ModelSim Altera software. For the control problem of the 4-leg 2-level inverter, we estimated computation times around $50 \mu \mathrm{s}$, which is appropriate for switching frequencies up to $10 \mathrm{kHz}$. Depending on the control objectives, the delay induced by the computing time should be taken into account by the control strategy. A solution is to perform a two-step prediction of the system states, and optimal control solutions will be applied only for the next control period.

\section{CONCLUSIONS}

In this paper, a new formulation of the control of a 4-leg 2level inverter was proposed. The problem was stated in terms of an optimization problem similar to the control allocation problem that was solved earlier in other applications, especial-

TABLE II

SIMULATION PARAMETERS

\begin{tabular}{ccc}
\hline \hline Symbol & Meaning & Values \\
\hline$T_{S}$ & Switching period & $100 \mu \mathrm{s}$ \\
$R, L$ & Load parameters & $0.5 \Omega, 10 \mathrm{mH}$ \\
$f$ & Fundamental frequency & $50 \mathrm{~Hz}$ \\
$E_{D C}$ & DC bus voltage & $400 \mathrm{~V}$ \\
\hline \hline
\end{tabular}

ly for flight control. The control objective was thus formulated as the distribution of the 3 reference voltages to the available redundant actuators, which consisted of the duty cycles of the switching cells. The control allocation theory presents an alternative solution to converter control taking a step back from the known electronic or geometric viewpoints. The available degree of freedom is used in an optimized manner as the voltage constraint set is fully taken into account. A major interest of this framework is its possible application to problems with large number of switching cells [31], [32]. In the context of this paper, however, where a small size, 4-leg 2-level inverter was considered, the main result was the development of an approach providing a whole range of solutions giving a maximal extension of the inverter linearity range, all under the umbrella of a single mathematical formulation. Investigations on optimization parameters revealed several possible choices with some corresponding to existing solutions, and others providing new approaches with distinct advantages, like OMIPWM [25] and ASPWM. An interesting feature of the analytic solutions obtained was that the resulting algorithms all reduced to carrier-based equivalent PWM methods. Moreover, they are applicable to the classic 3-leg 2-level inverter connected to a threephase balanced load [25].

As the control allocation method optimizes the use of available resources with a general problem formulation, the approach seems promising for implementing a fault tolerant $c a-$ pability. However, this feature was not studied in this paper as the 4-leg 2-level inverter is not adequate for a meaningful investigation of redundancy.

\section{REFERENCES}

[1] D. G. Holmes and T. A. Lipo, Pulse Width Modulation for Power Converters: principles and practice, New York: Wiley-IEEE Press, 2003.

[2] J. Holtz, "Pulsewidth Modulation for Electronic Power Conversion," Proc. IEEE, vol. 82, no. 8, pp. 1194-1214, Aug. 1994.

[3] S. R. Bowes, "New sinusoidal pulsewidth-modulated invertor," Proc. Inst. Elect. Eng., vol. 122, pp. 1279-1285, Nov. 1975.

[4] J. Holtz, P. Lammert and W. Lotzkat, "High-Speed Drive System with Ultrasonic MOSFET PWM Inverter and Single-Chip Microprocessor Control," IEEE Trans. Indus. Appl., vol. IA-23, no. 6, pp. 1010-1015, Nov. 1987.

[5] H. W. Van der Broeck, H.-C. Skudelny and G. V. Stanke, "Analysis and Realization of a Pulsewidth Modulator Based on Voltage Space Vectors," IEEE Trans. Indus. Appl., vol. 24, no. 1, pp. 142-150, Jan.-Feb. 1988.

[6] D. G. Holmes, "The significance of zero space vector placement for carrier-based PWM schemes,", IEEE Trans. Indus. Appl., vol. 32, no. 5, pp. 1122-1129, 1996. 
[7] S. R. Bowes and Y.-S. Lai, "The relationship between space-vector modulation and regular-sampled PWM," IEEE Trans. Indus. Electron., vol. 44, no. 5, pp. 670-679, Oct. 1997.

[8] C. B. Jacobina, A. M. N. Lima, E. R. C. da Silva, R. N. C. Alves and P. F. Seixas, "Digital scalar pulse-width modulation: a simple approach to introduce nonsinusoidal modulating waveforms," IEEE Trans. Power Electron., vol. 16, no. 3, pp. 351-359, May 2001.

[9] E. R. C. da Silva, E. C. dos Santos, and C. B. Jacobina, "Pulsewidth Modulation Strategies," IEEE Indus. Electron. Mag., vol. 5, no. 2, pp. 37-45, June 2011.

[10] J.-H. Kim and S.-K. Sul, "A Carrier-Based PWM Method for ThreePhase Four-Leg Voltage Source Converters," IEEE Trans. Power Electron., vol. 19, no. 1, pp. 66-75, Jan. 2004

[11] N.-Y. Dai, M.-C. Wong, F. Ng and Y.-D. Han, "A FPGA-Based Generalized Pulse Width Modulator for Three-Leg Center-Split and Four-Leg Voltage Source Inverters," IEEE Trans. Power Electron., vol. 23, no. 3, pp. 1472-1484, May 2008.

[12] X. Li, Z. Deng, Z. Chen and Q. Fei, "Analysis and Simplification of Three-Dimensional Space Vector PWM for Three-Phase Four-Leg Inverters," IEEE Trans. Indus. Electron., vol. 58, no. 2, pp. 450-464, Feb. 2011.

[13] G. Buja and G. Indri, "Improvement of Pulse Width Modulation Techniques," Archiv für Elektrotechnik, vol. 57, pp. 281-289, 1975.

[14] J. A. Houldsworth and D. A. Grant, "The Use of Harmonic Distortion to Increase the Output Voltage of a Three-Phase PWM Inverter," IEEE Trans. Indus. Appl., vol. IA-20, no. 5, pp. 1224-1228, Sept.-Oct. 1984.

[15] S. Ogasawara, H. Akagi, and A. Nabae, "A novel PWM scheme of voltage source inverters based on space vector theory," Archiv für Elektrotechnik, vol. 74, no. 1, pp. 33-41, Jan. 1990.

[16] A. M. Hava, R. J. Kerkman, and T. A. Lipo, "A high-performance generalized discontinuous PWM algorithm," IEEE Transactions on Industry Applications, vol. 34, no. 5, pp. 1059-1071, Sep. 1998.

[17] J. W. Kolar, H. Ertl and F. C. Zach, "Minimizing the current harmonics RMS value of three-phase PWM converter systems by optimal and suboptimal transition between continuous and discontinuous modulation," in 22nd Annual IEEE PESC, 1991, pp. 372-381.

[18] J. Holtz and B. Beyer, "Optimal pulsewidth modulation for AC servos and low-cost industrial drives," IEEE Trans. Indus. Appl., vol. 30, no. 4, pp. 1039-1047, Jul. 1994

[19] R. Zhang, V. H. Prasad, D. Boroyevich and F. C. Lee, "ThreeDimensional Space Vector Modulation for Four-Leg Voltage-Source Converters," IEEE Trans. Power Electron., vol. 17, no. 3, pp. 314-326, May 2002.

[20] O. Ojo and P. M. Kshirsagar, "Concise Modulation Strategies for FourLeg Voltage Source Inverters," IEEE Trans. Power Electron., vol. 19, no. 1, pp. 46-53, Jan. 2004.

[21] M. A. Perales, M. M. Prats, R. Portillo, J. L. Mora, J. I. Leon and L. G. Franquelo, "Three-Dimensional Space Vector Modulation in abc Coordinates for Four-Leg Voltage Source Converters," IEEE Power Electron. Letters, vol. 1, no. 4, pp. 104-109, Dec. 2003.

[22] M. Bodson, "Evaluation of Optimization Methods for Control Allocation," Journal of Guidance, Control and Dynamics, vol. 25, no. 4, pp. 703-711, July-Aug. 2002.

[23] T. A. Johansen and T. I. Fossen, "Control Allocation-A survey," Automatica, vol. 49, pp. 1087-1103, 2013.

[24] A. Bouarfa, M. Fadel, M. Bodson and J. Lin, "A New Control Allocation Method for Power Converters and its Application to the Four-Leg Two-Level Inverter," in IEEE $23^{\text {rd }}$ Mediterranean Conf. Control Autom. (MED), Torremolinos, Spain, pp. 1020-1026, 16-19 June 2015.

[25] A. Bouarfa, M. Fadel, and M. Bodson, "A new PWM method for a 3phase 4-leg inverter based on the injection of the opposite median reference voltage," in 2016 Int. Symp. Power Electron. Electr. Drives Autom. Motion (SPEEDAM), Anacapri, Italy, pp. 791-796, June 2016.

[26] C. G. Small, "A Survey of Multidimensional Medians," International Statistical Review, vol. 58, no. 3, pp. 263-277, 1990.

[27] J. A. Hanley, L. Joseph, R. W. Platt, M. K. Chung, and P. Belisle, "Visualizing the Median as the Minimum-Deviation Location," The American Statistician, vol. 55, no. 2, pp. 150-152, May 2001.

[28] D. W. Stroock, Probability Theory: An Analytic View. Cambridge University Press, 2010

[29] J.-B. Hiriart-Urruty and C. Lemaréchal, Fundamentals of Convex Analysis. Berlin, Heidelberg: Springer Berlin Heidelberg, 2001.
[30] Z. Drezner and H. W. Hamacher, Facility Location: Applications and Theory. Springer Science \& Business Media, 2004

[31] A. Bouarfa, M. Bodson, and M. Fadel, "A fast active-balancing method for the 3-phase multi-level flying capacitor inverter derived from control allocation theory," in $201720^{\text {th }}$ IFAC World Congress, Toulouse, France, July 2017.

[32] A. Bouarfa, M. Fadel, and M. Bodson, "A numerical optimization method using the simplex algorithm for control of modular multilevel converters," in ELECTRIMACS 2017, Toulouse, France, July 2017.

[33] S. Bayliss, C. Bouganis, G. A. Constantinides, and W. Luk, "An FPGA implementation of the simplex algorithm," in 2006 IEEE International Conference on Field Programmable Technology, 2006, pp. 49-56.

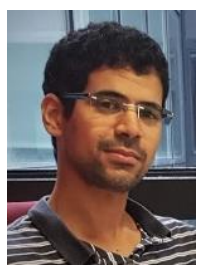

Abdelkader Bouarfa was born near Paris, France, in 1991. He received the degree of engineer in electrical engineering and automation from the Ecole Nationale Supérieure d'Electrotechnique, d'Electronique, d'Informatique, d'Hydraulique et des Télécommunications (ENSEEIHT), engineer school member of the National Polytechnic Institute of Toulouse (INPT), Toulouse, France, in 2014.

$\mathrm{He}$ is currently preparing a Ph.D. thesis in control of power converters at the Laboratory of Plasma and Energy Conversion, LAPLACE, Université de Toulouse, mixed unit of research CNRS-INPTUPS. His research interests encompass control of energy conversion systems.

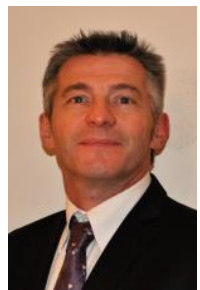

Maurice Fadel (M'09) was born in Toulouse, France. He got the $\mathrm{PhD}$ degree at the Institut National Polytechnique de Toulouse in 1988, in the domain of the Control in Electric Engineering.

He is currently a Professor in the Ecole Nationale Supérieure d'Electrotechnique, d'Electronique, d'Informatique, d'Hydraulique et des Télécommunications of Toulouse (ENSEEIHT). In 1985 he as integrated the Laboratory of Electrotechnics and Industrial electronics (LEEI), mixed unit of research (CNRS-INPT-Univ. Toulouse III). He was leading of the LEEI laboratory in 2005. From January 2007 until December 2015 he was Deputy Director of the Laboratoire Plasma et Conversion d'Energie (Laboratory of Plasma and Energy Conversion, LAPLACE). This laboratory count about hundred permanent researchers, more than hundred $\mathrm{PhD}$ students covering the continuum of specialty, materials, plasmas and systems to the service of the conversion and the treatment of the electric energy. The field of scientific interest of Pr. Maurice FADEL concerns the modeling and the control of the electric systems more especially of the synchronous machine, the control law of the static converters with the help of direct predictive controls approach and the definition of control strategies for cooperative systems.

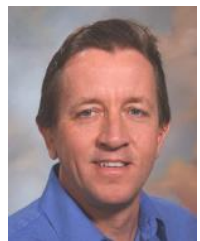

Marc Bodson (F'06) received a Ph.D. degree in Electrical Engineering and Computer Science from the University of California, Berkeley, in 1986. He obtained two M.S. degrees - one in Electrical Engineering and Computer Science and the other in Aeronautics and Astronautics - from the Massachusetts Institute of Technology, Cambridge MA, in 1982. In 1980, he received the degree of Ingénieur Civil Mécanicien et Electricien from the Université Libre de Bruxelles, Belgium. His research interests are in adaptive control, with applications to electromechanical systems and aerospace.

Currently, he is a Professor of Electrical \& Computer Engineering at the University of Utah in Salt Lake City. He was Chair of the department between 2003 and 2009, and he was an Assistant Professor and an Associate Professor at Carnegie Mellon University, Pittsburgh, PA, between 1987 and 1993. He was a Belgian American Educational Foundation Fellow in 1980 and a Lady Davis Fellow at the Technion, Haifa, Israel, in 1990. He is coauthor, with S. Sastry, of the book Adaptive Control: Stability, Convergence, and Robustness, published by Prentice-Hall in 1989 and reprinted by Dover in 2011.

Professor Bodson was the Editor-in-Chief of IEEE Trans. on Control Systems Technology from 2000 to 2003. He was elected Fellow of the IEEE in 2006, and Associate Fellow of the American Institute of Aeronautics and Astronautics in 2013. He received the Engineering Educator of the Year award from the Utah Engineers Council in 2007. 